\title{
Single-cell RNA-seq revealed the role of Alkbh5 in reproduction
}

\author{
Xiaozhong Shen ${ }^{1}$, Gangcai Xie ${ }^{1 *}$ \\ 1 Medical school, Nantong University, Nantong, China ;
}

* Corresponding author

Gangcai Xie, Ph.D

Institute of Reproductive Medicine, Medical School, Nantong University

Qixiu Road 19, Nantong, China, 226001

Email: gangcai@ntu.edu.cn

This manuscript has been withdrawn by the authors as it was submitted and made public without the full consent of all the authors. Therefore, the authors do not wish this work to be cited as reference for the project. If you have any questions, please contact the corresponding author 